\title{
A short chiral route towards polyhydroxylated indolizidines and quinolizidines
}

Steven H. L. Verhelst, Baltasar Paez Martinez, Mattie S. M. Timmer, Gerrit Lodder, Gijsbert A. van der Marel, Herman S. Overkleeft*, Jacques H. van Boom*.

Leiden Institute of Chemistry, Gorlaeus Laboratories, Leiden University, PO Box 9502, 2300 RA, Leiden, The Netherlands.

E-mail: j.boom@chem.leidenuniv.nl

\section{SUPPORTING INFORMATION}

\section{Contents}

$\begin{array}{ll}\text { General methods and materials } & \text { S2 }\end{array}$

$\begin{array}{ll}\text { Spectra Compound 5b } & \text { S3 }\end{array}$

$\begin{array}{ll}\text { Spectra Compound } 7 & \text { S5 }\end{array}$

$\begin{array}{ll}\text { Spectra Compound } \mathbf{1 3} & \text { S7 }\end{array}$

$\begin{array}{ll}\text { Spectra Compound } \mathbf{1 5} & \text { S9 }\end{array}$

$\begin{array}{ll}\text { Spectra Compound } 16 & \text { S11 }\end{array}$

$\begin{array}{lr}\text { Spectra Compounds 8a/9a } & \text { S13 }\end{array}$

$\begin{array}{lr}\text { Spectra Compounds 8b/9b } & \text { S17 }\end{array}$

$\begin{array}{lr}\text { Spectra Compounds 8c/9c } & \text { S21 }\end{array}$

$\begin{array}{lr}\text { Spectra Compounds 8d/9d } & \text { S25 }\end{array}$ 


\section{Experimental}

\section{General Methods and Materials.}

A successful outcome of the Vasella-Barbier tandem reaction is largely dependent on the quality of the zinc. Prior to reactions, zinc dust (Acros) was activated with $2 \mathrm{M} \mathrm{HCl}$, washed successively with water, ethanol and diethyl ether and dried in vacuo. 


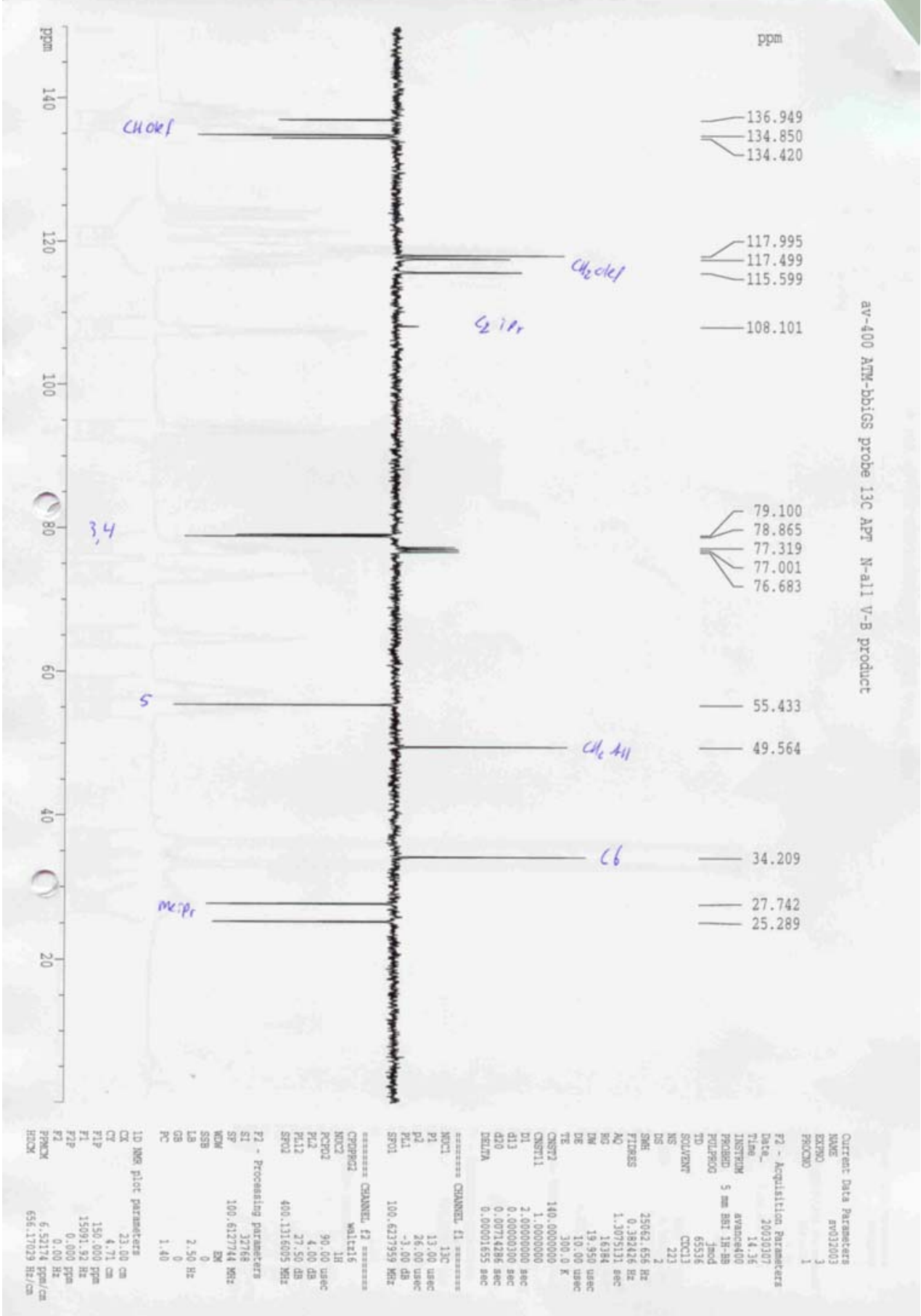

$100 \mathrm{MHz}$ C13 spectrum compound $\mathbf{5 b}$

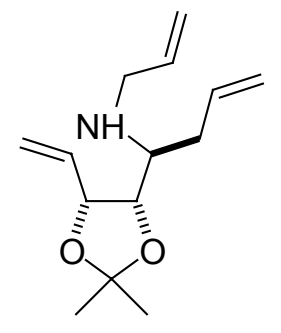




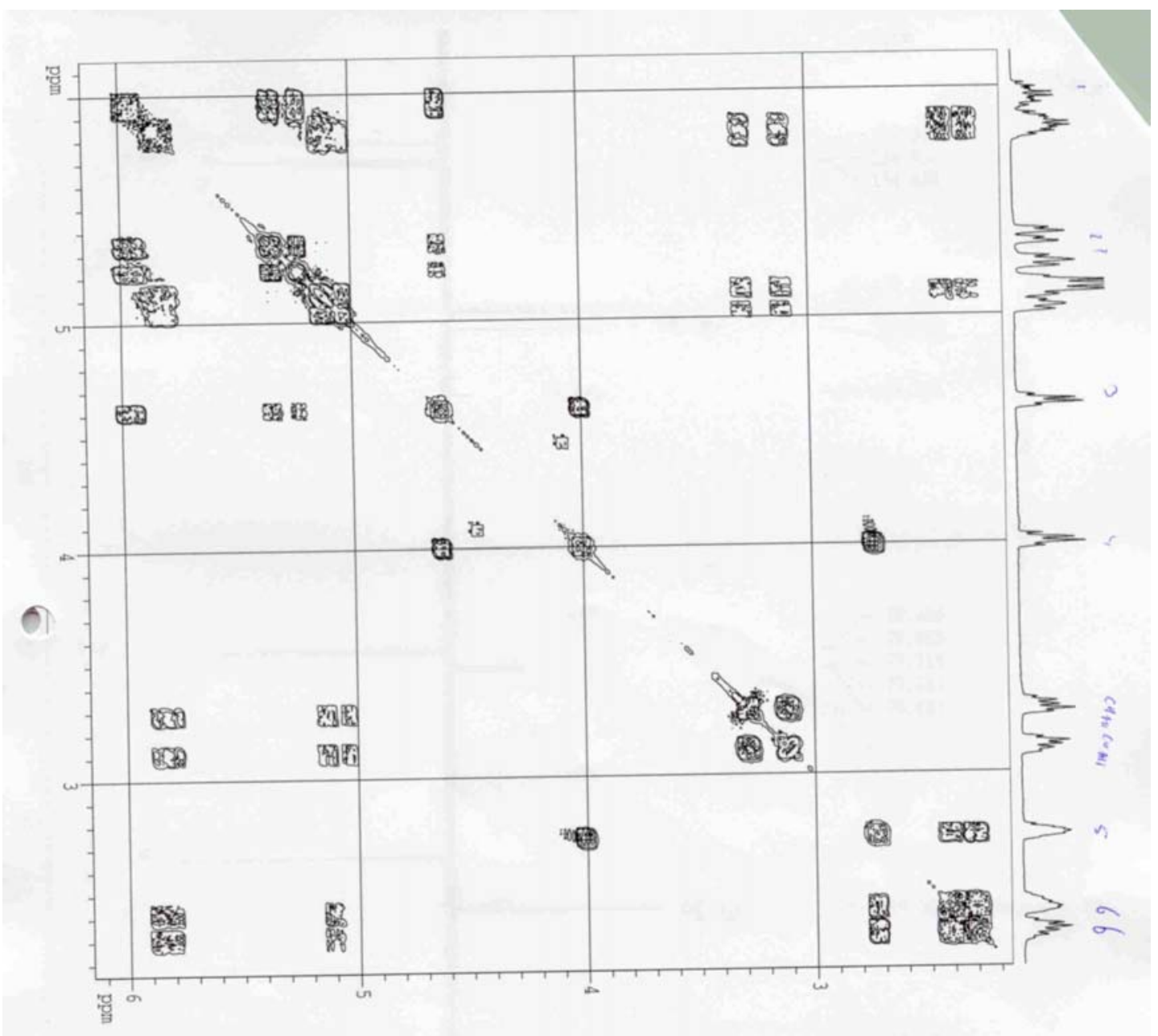

$400 \mathrm{MHz}$ COSY spectrum of compound $\mathbf{5 b}$

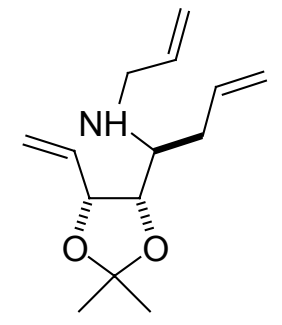




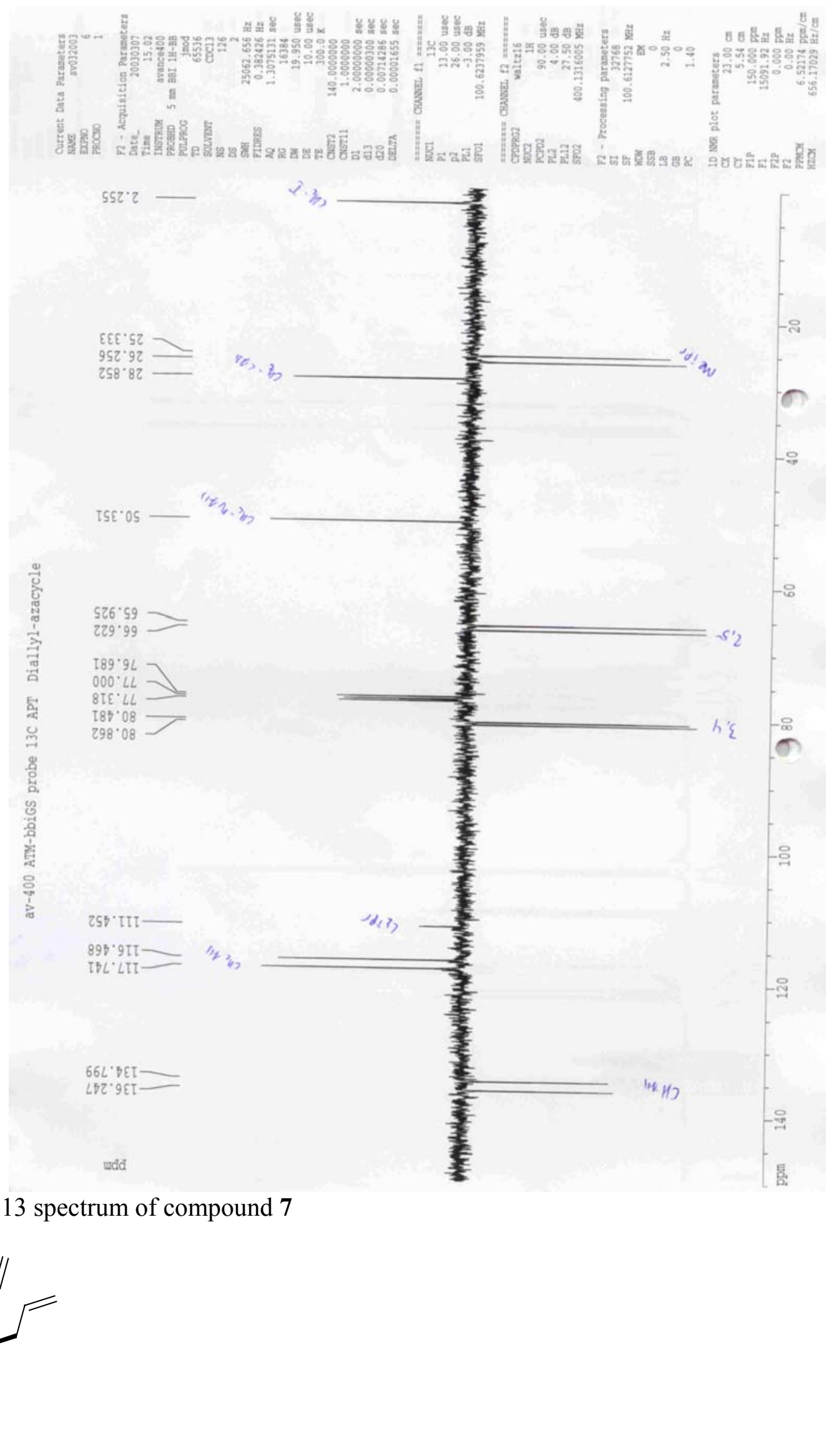




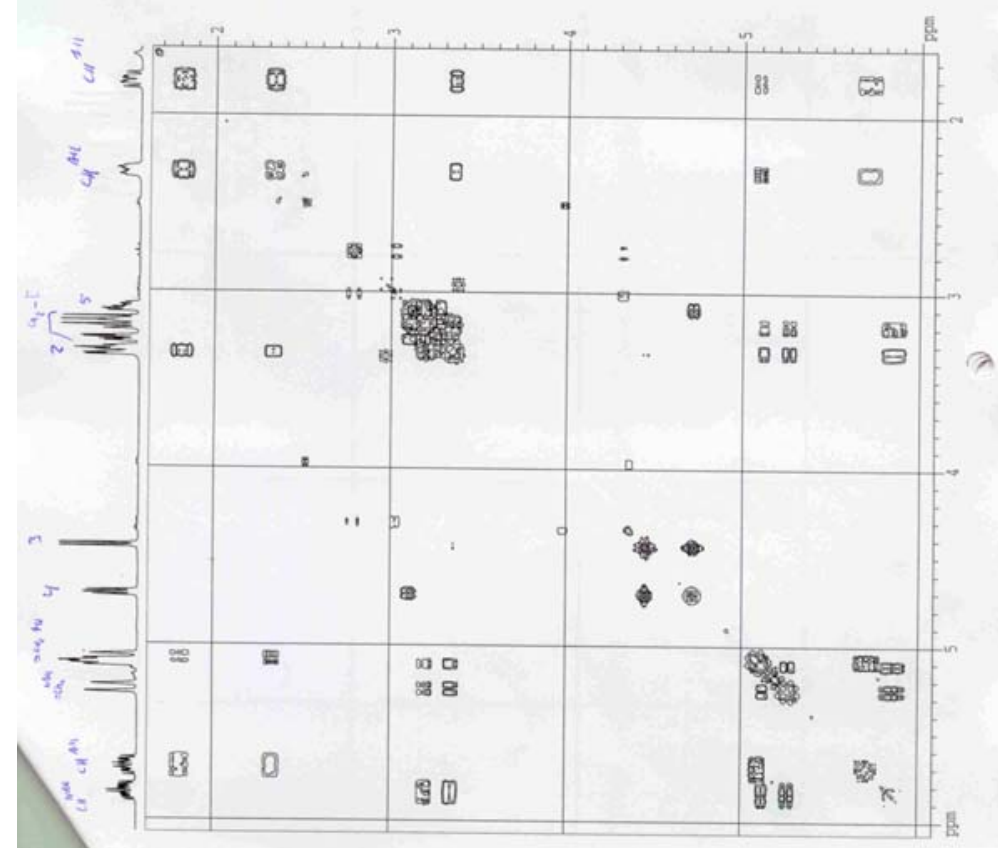

$400 \mathrm{MHz}$ COSY spectrum of compound 7

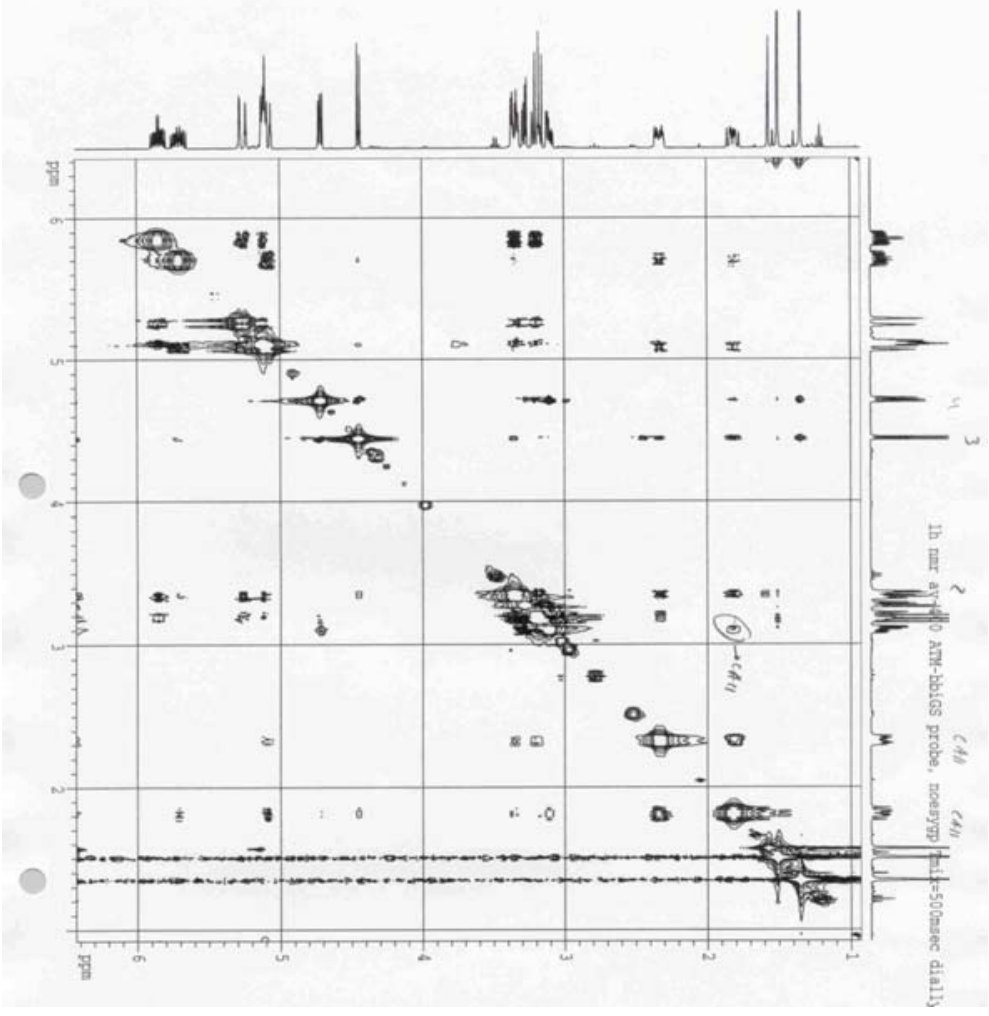

$400 \mathrm{MHz}$ NOESY spectrum of compound 7 


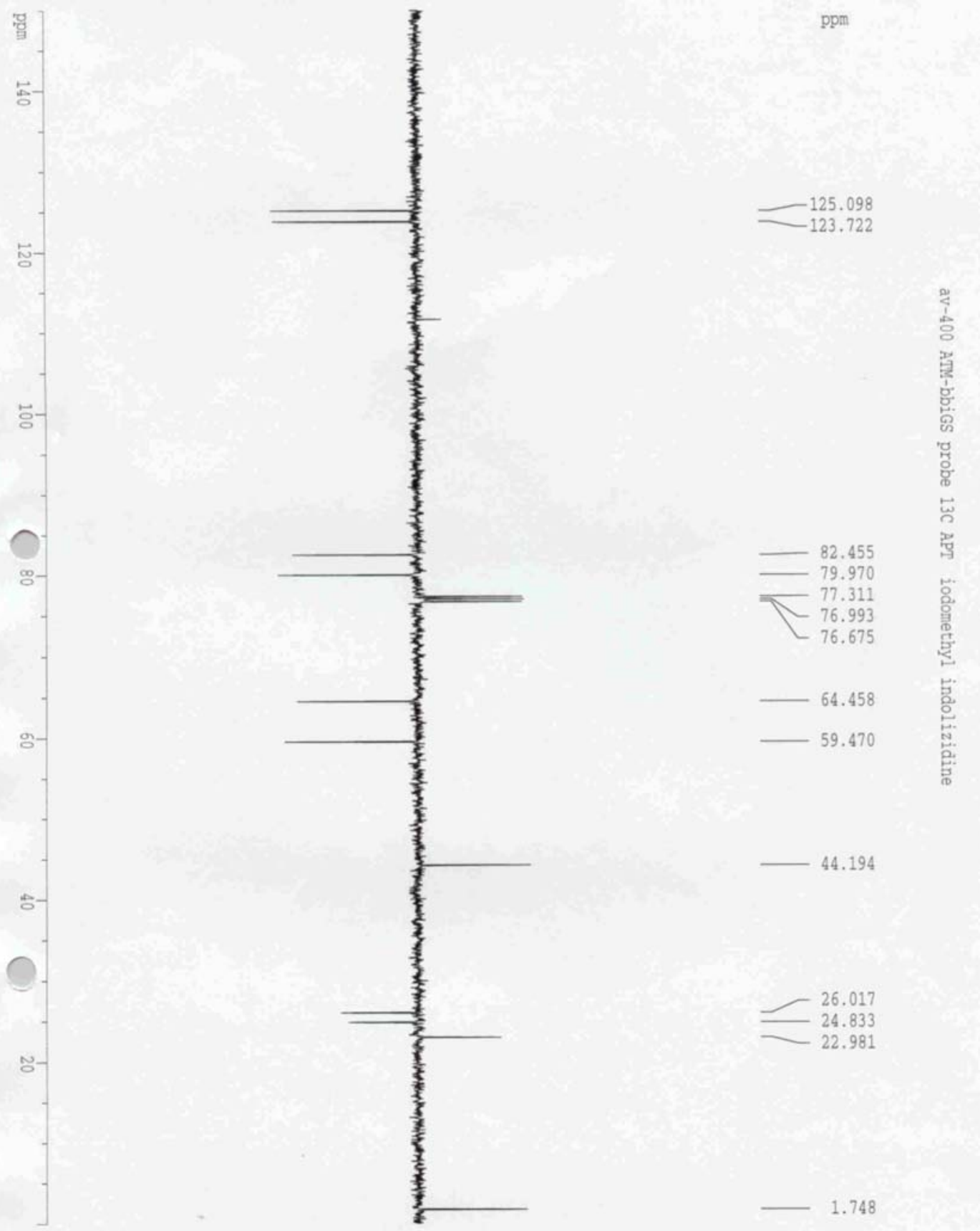

$100 \mathrm{MHz}$ C13 spectrum of compound $\mathbf{1 3}$<smiles>CC1(C)[CH][C@H]2CC=CCN([C@H]3CC=CCN3)[C@H]2O1</smiles> 


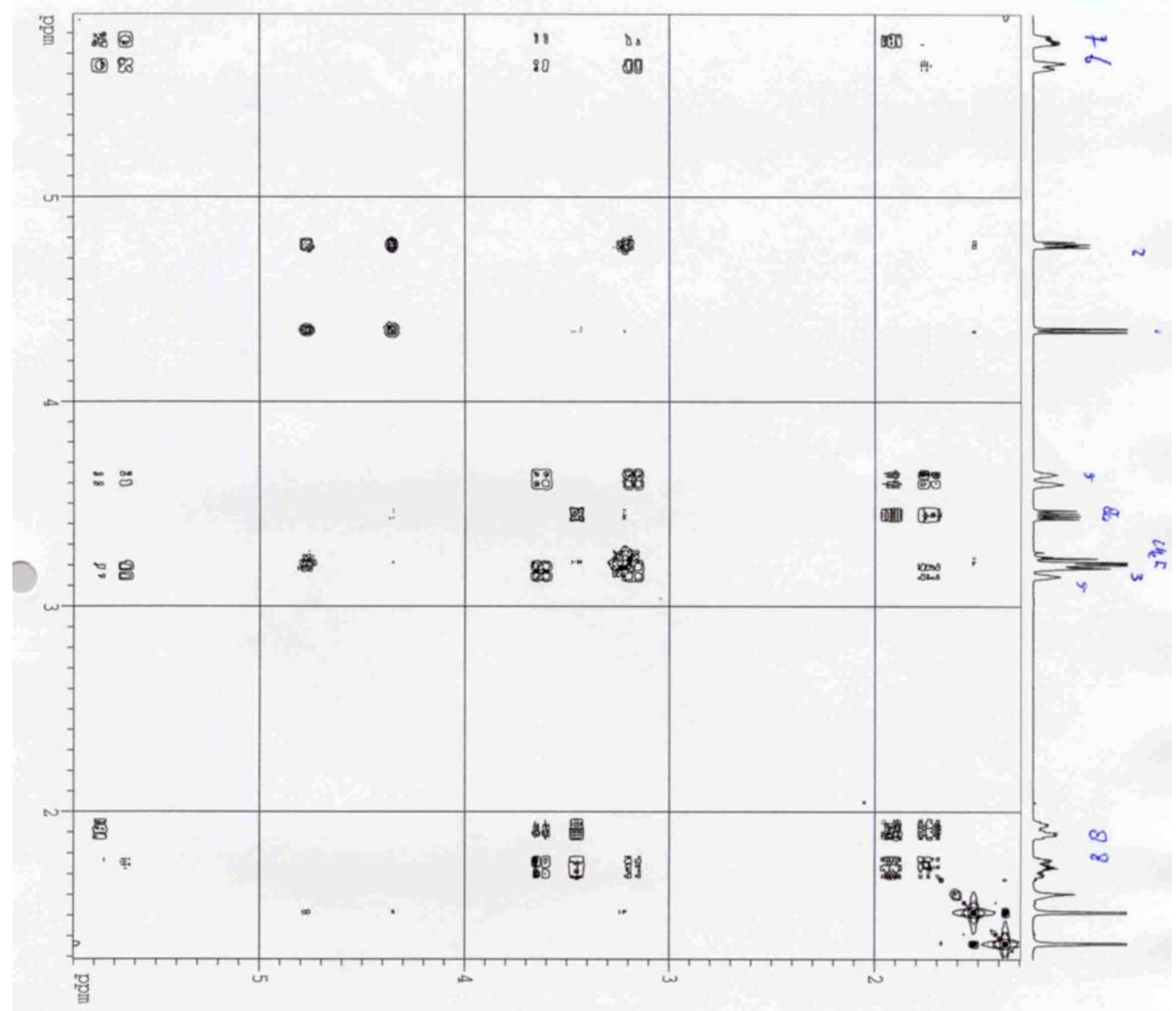

$400 \mathrm{M} H z$ COSY spectrum of compound $\mathbf{1 3}$<smiles>CC1(C)O[C@H]2[C@@H](CI)N3CC=CC[C@H]3[C@H]2O1</smiles> 

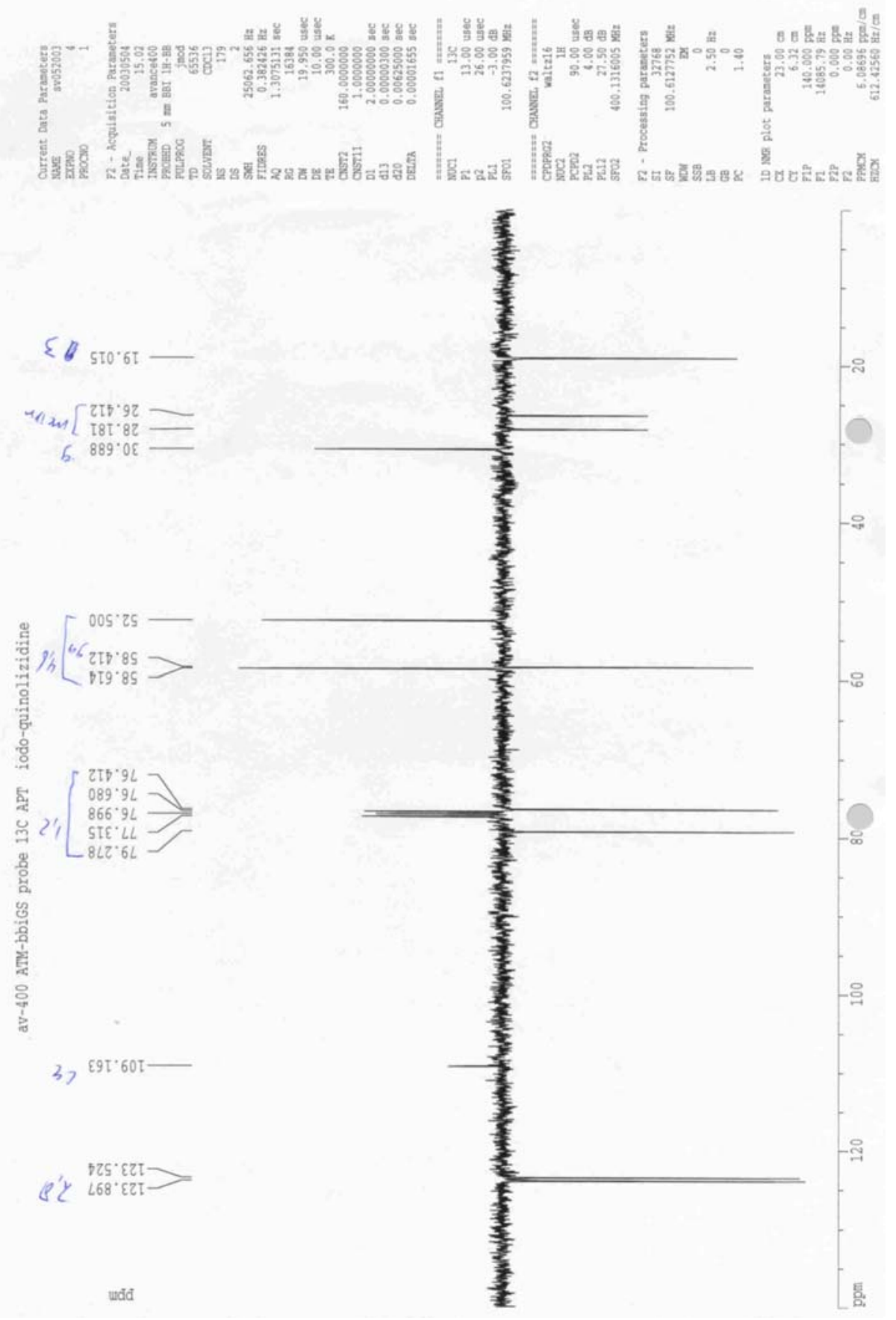

$100 \mathrm{MHz}$ C13 spectrum of compound $\mathbf{1 5}$<smiles>CC1(C)O[C@H]2[C@@H]3CC=CCN3C[C@H](I)[C@@H]2O1</smiles> 


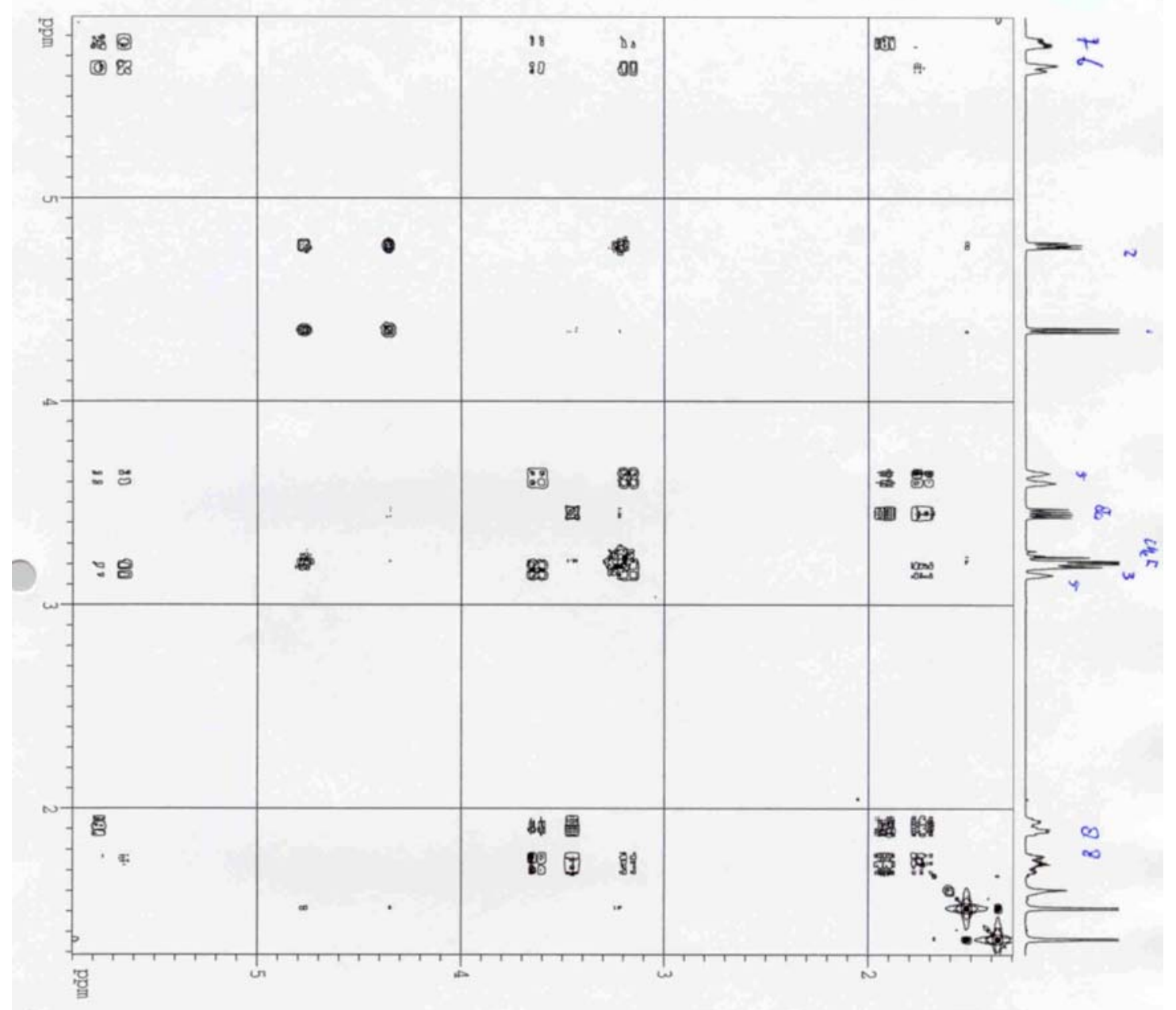

$400 \mathrm{MHz}$ COSY spectrum of compound $\mathbf{1 5}$<smiles>CN1CC=CC[C@H]2[C@@H]1OC(C)(C)[C@@H]2I</smiles> 


$$
1
$$




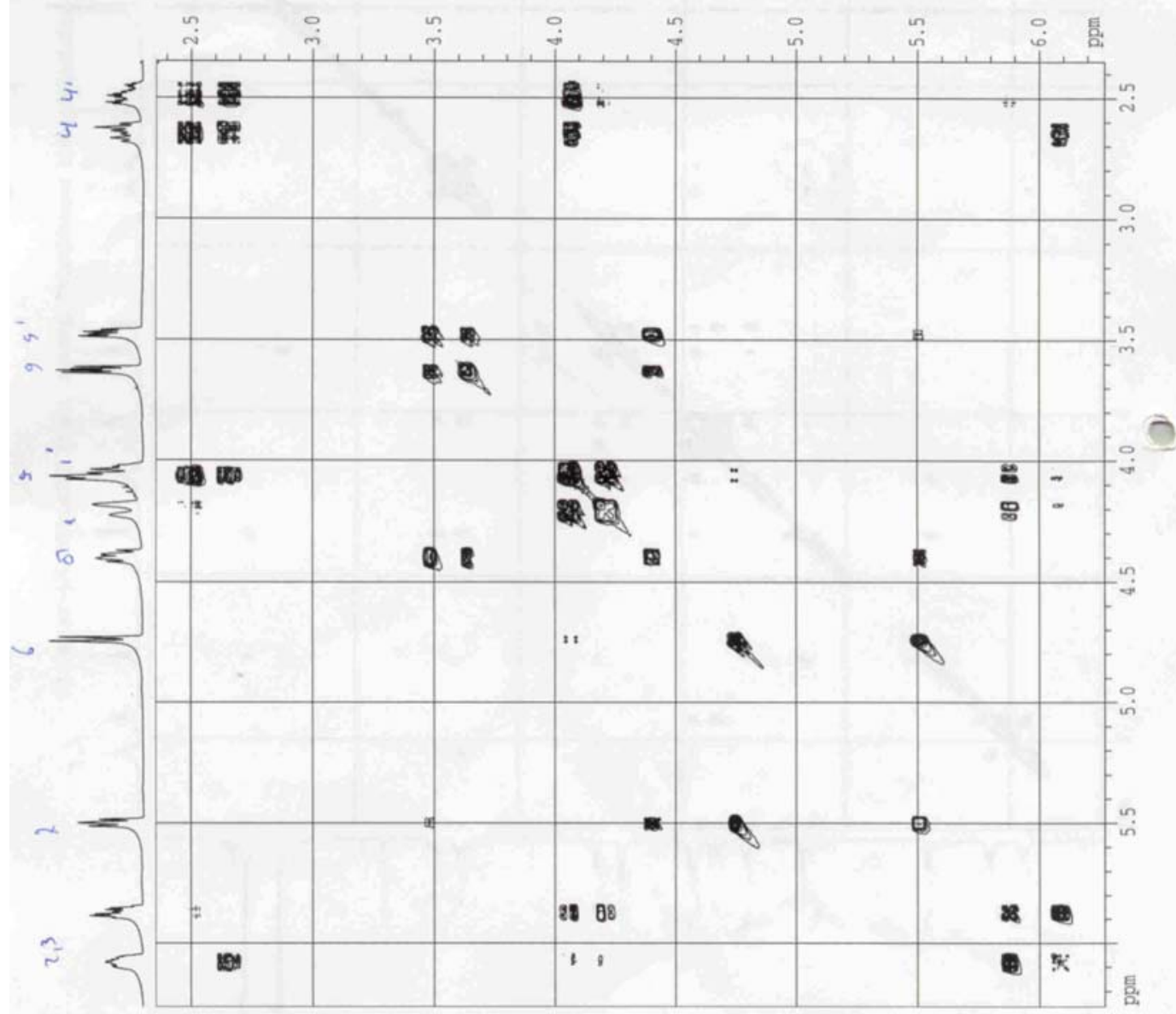

$400 \mathrm{MHz}$ COSY spectrum of intermediate aziridinium ion $\mathbf{1 6}$

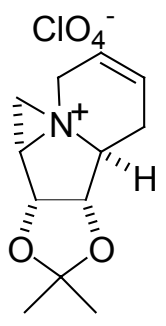




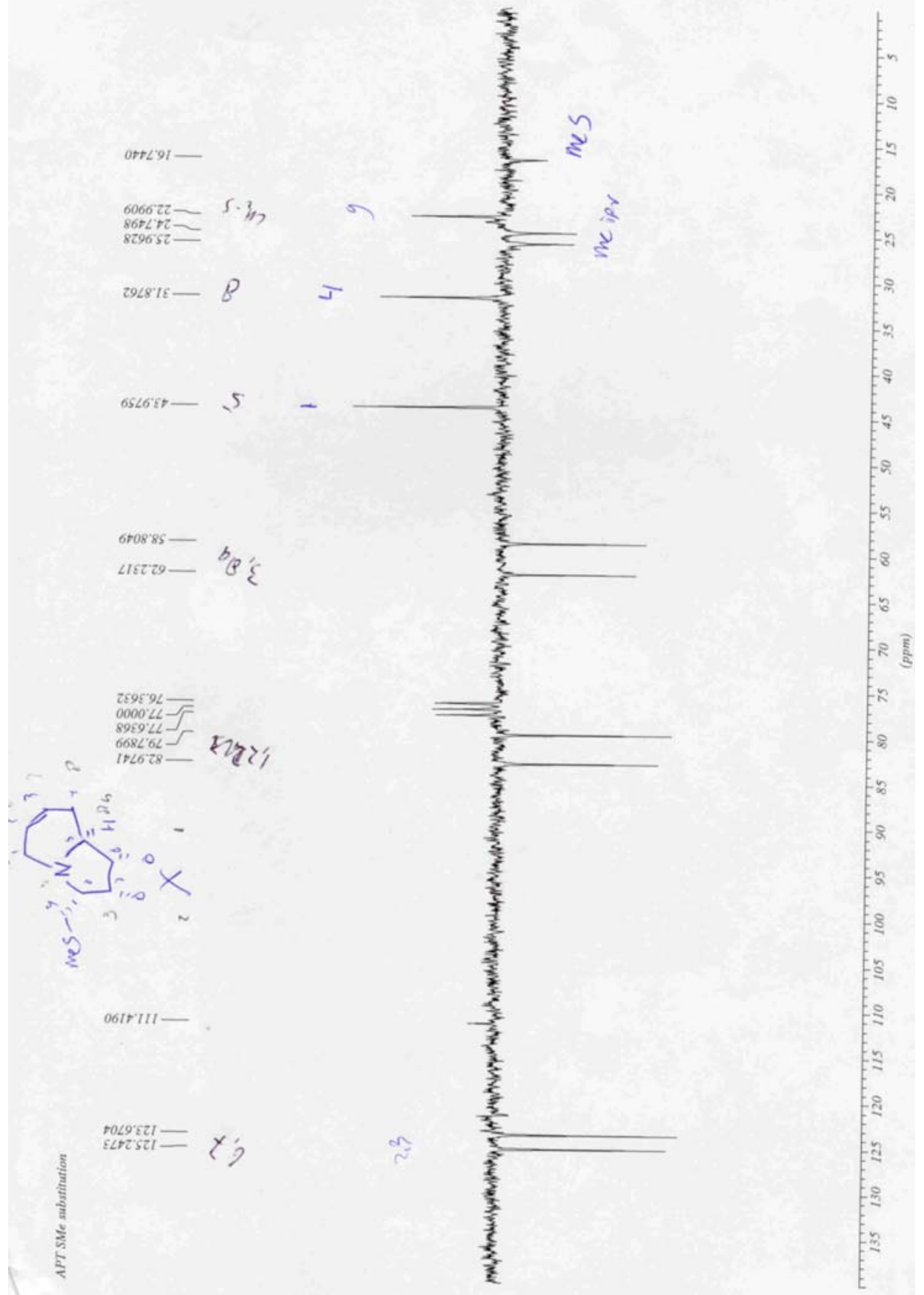

50.1 MHz C13 spectrum of compound 8a<smiles>CC[C@H]1[C@@H]2OC(C)(C)O[C@H]2[C@H]2CC=CCN12</smiles> 


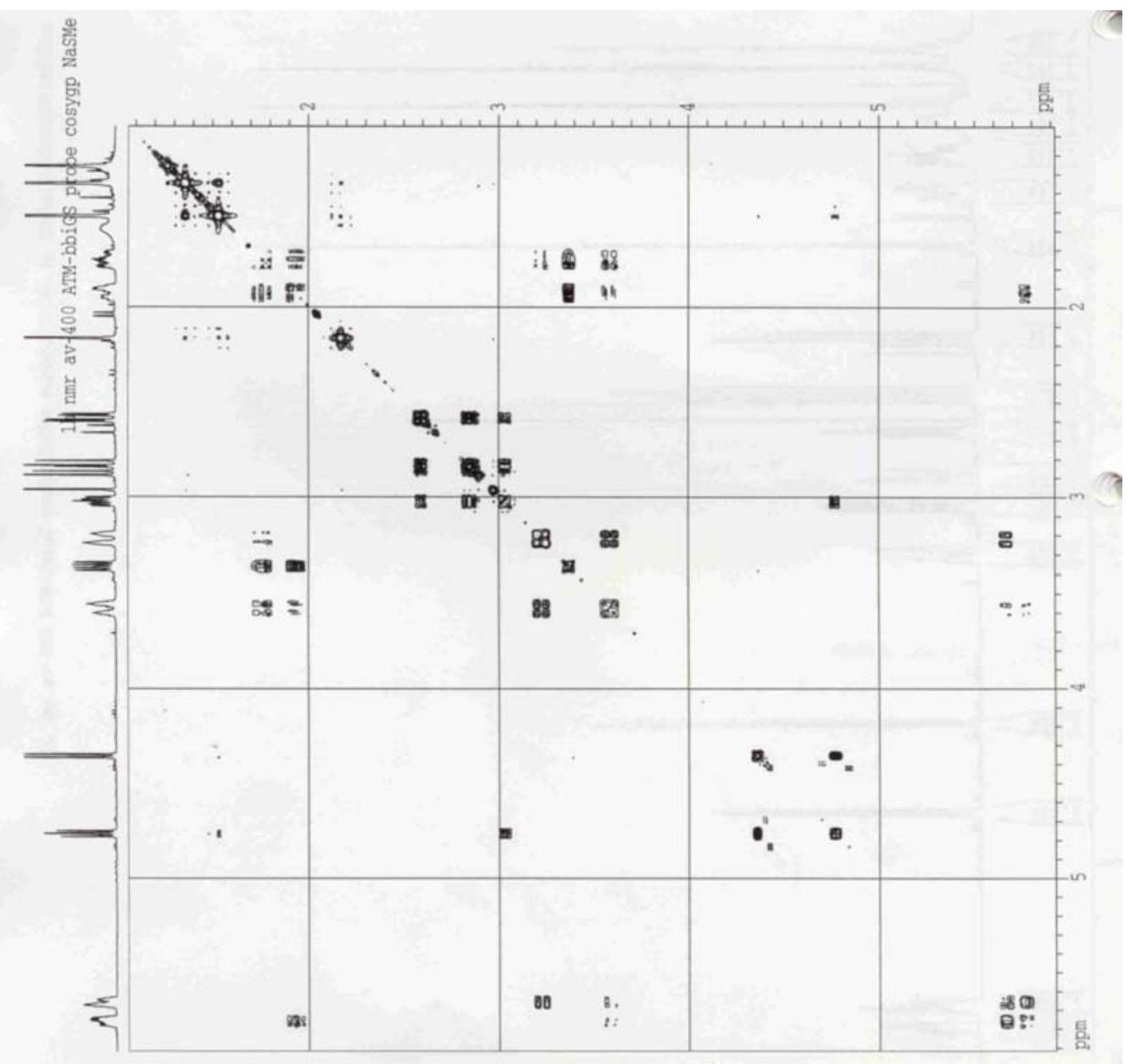

$400 \mathrm{MHz}$ COSY spectrum of compound 8a<smiles>CC[C@H]1[C@@H]2OC(C)(C)O[C@H]2[C@H]2CC=CCN12</smiles> 

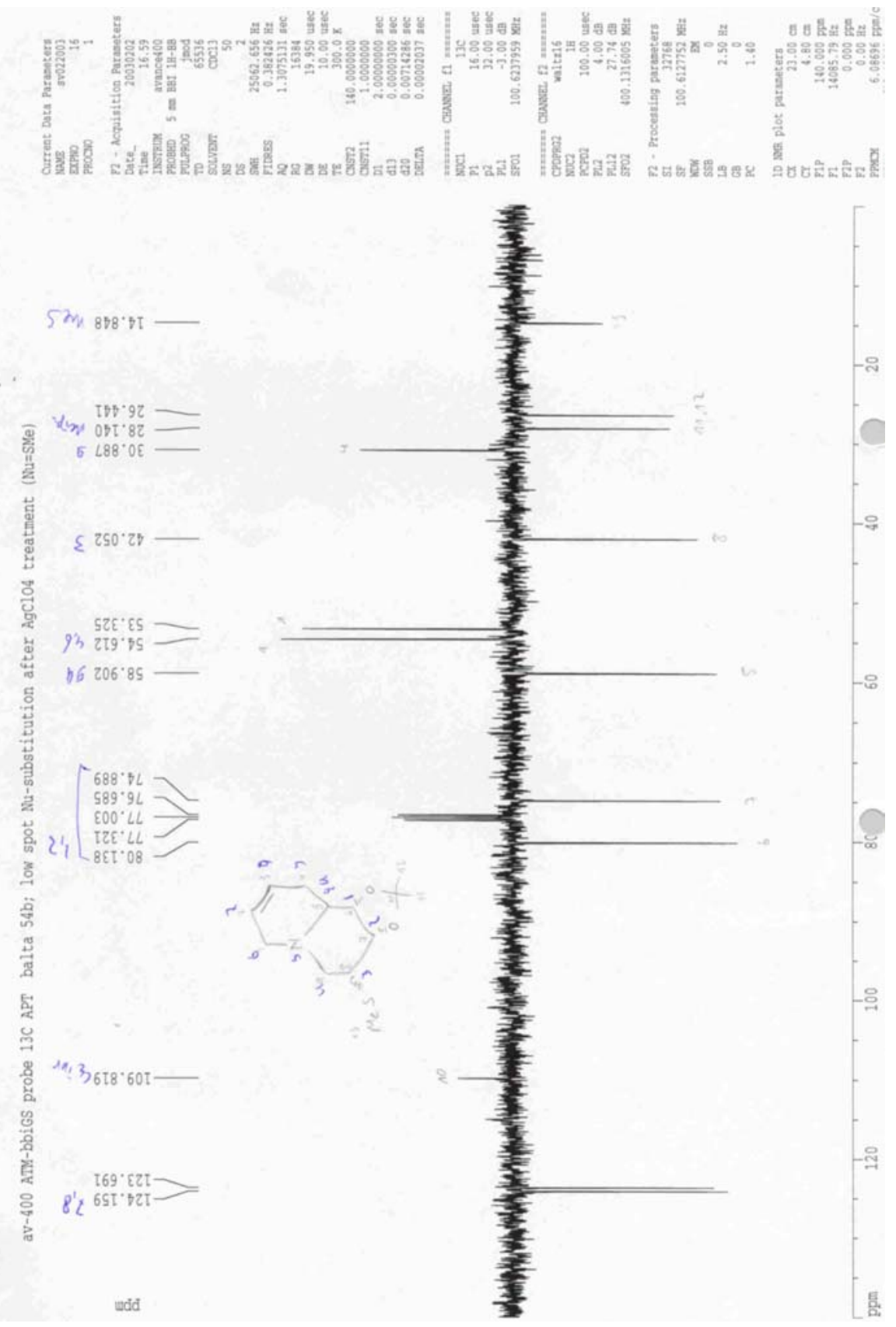

$100 \mathrm{MHz}$ C13 spectrum of compound 9a<smiles>CC=CCN1CC(C)(C)O[C@H]2CC(C)(C)O[C@@H]21</smiles> 


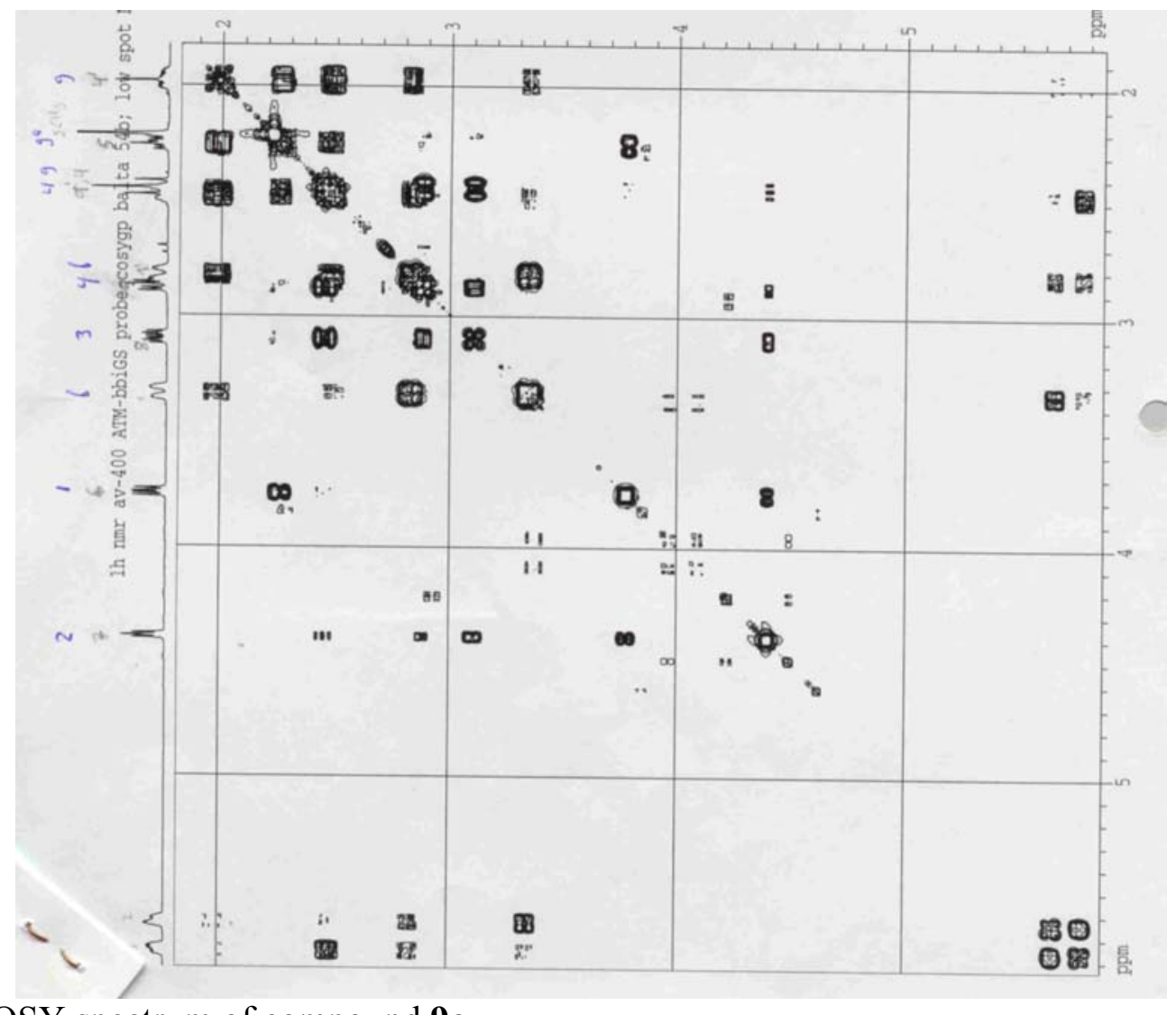

$400 \mathrm{MHz}$ COSY spectrum of compound 9a<smiles>CC=CCN1CC(C)(C)O[C@H]2CC(C)(C)O[C@@H]21</smiles> 


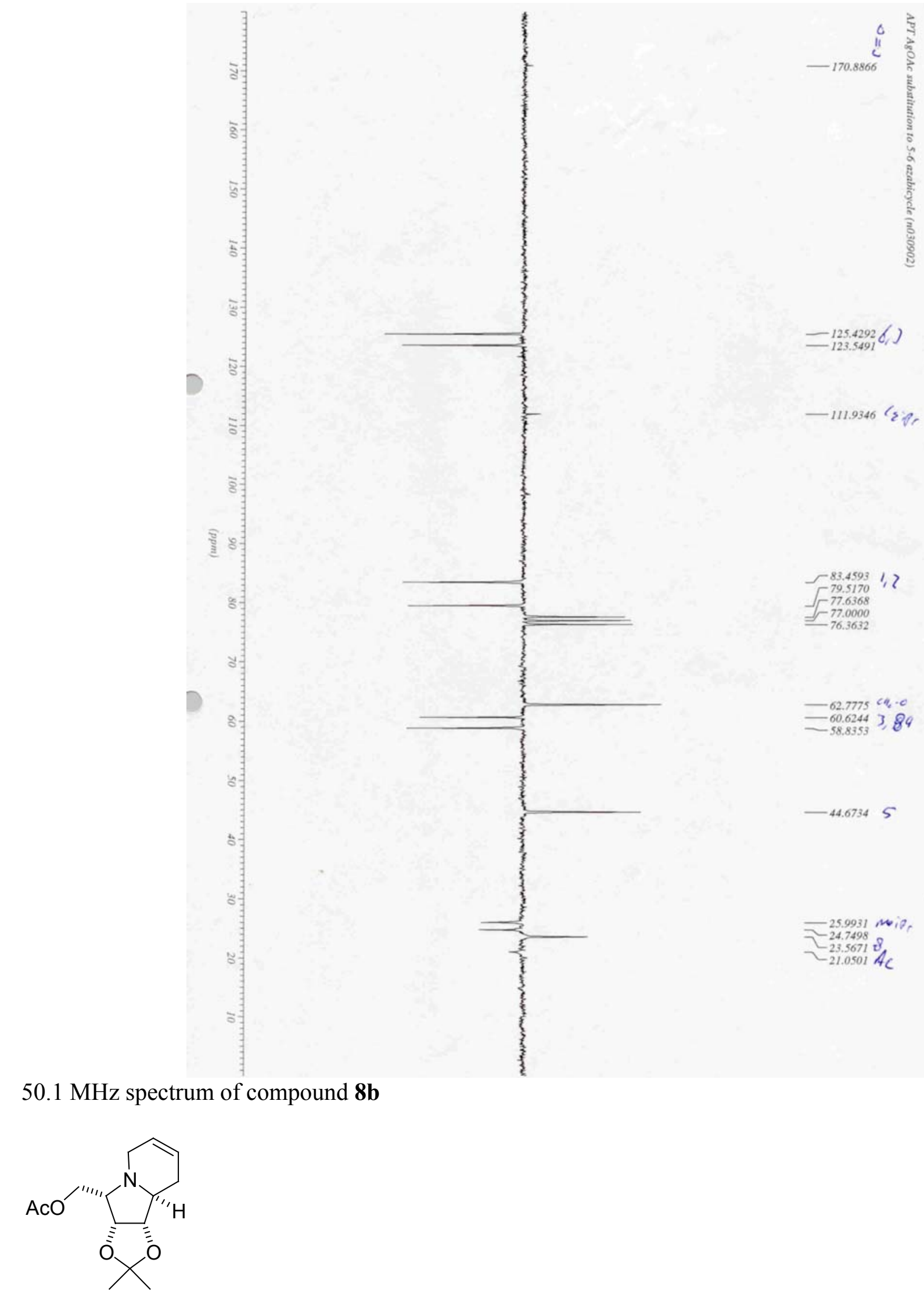




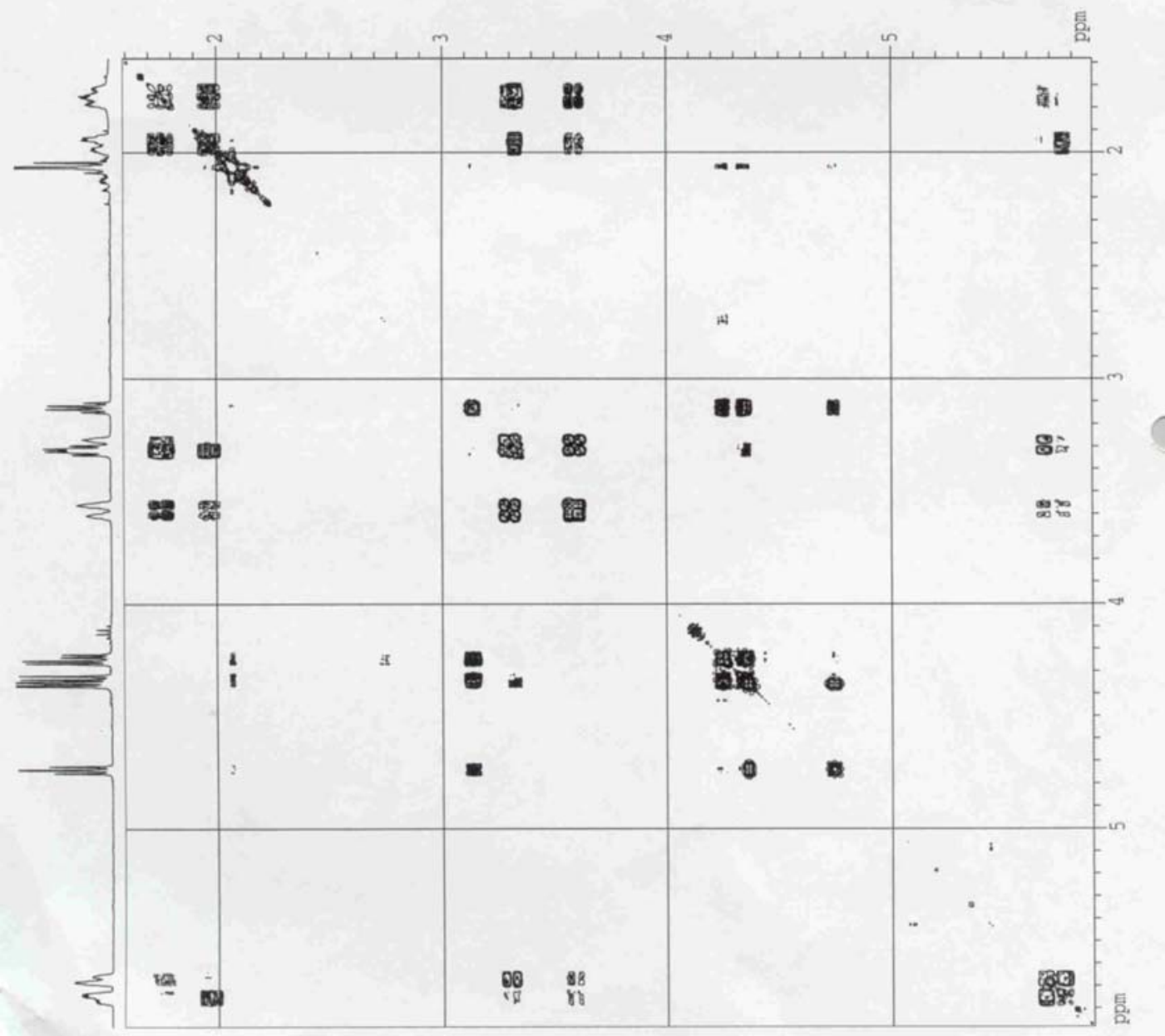

$400 \mathrm{MHz}$ COSY spectrum of compound $\mathbf{8 b}$<smiles>CC(=O)C[C@@H]1[C@@H]2OC(C)(C)O[C@H]2[C@H]2CC=CCN21</smiles> 


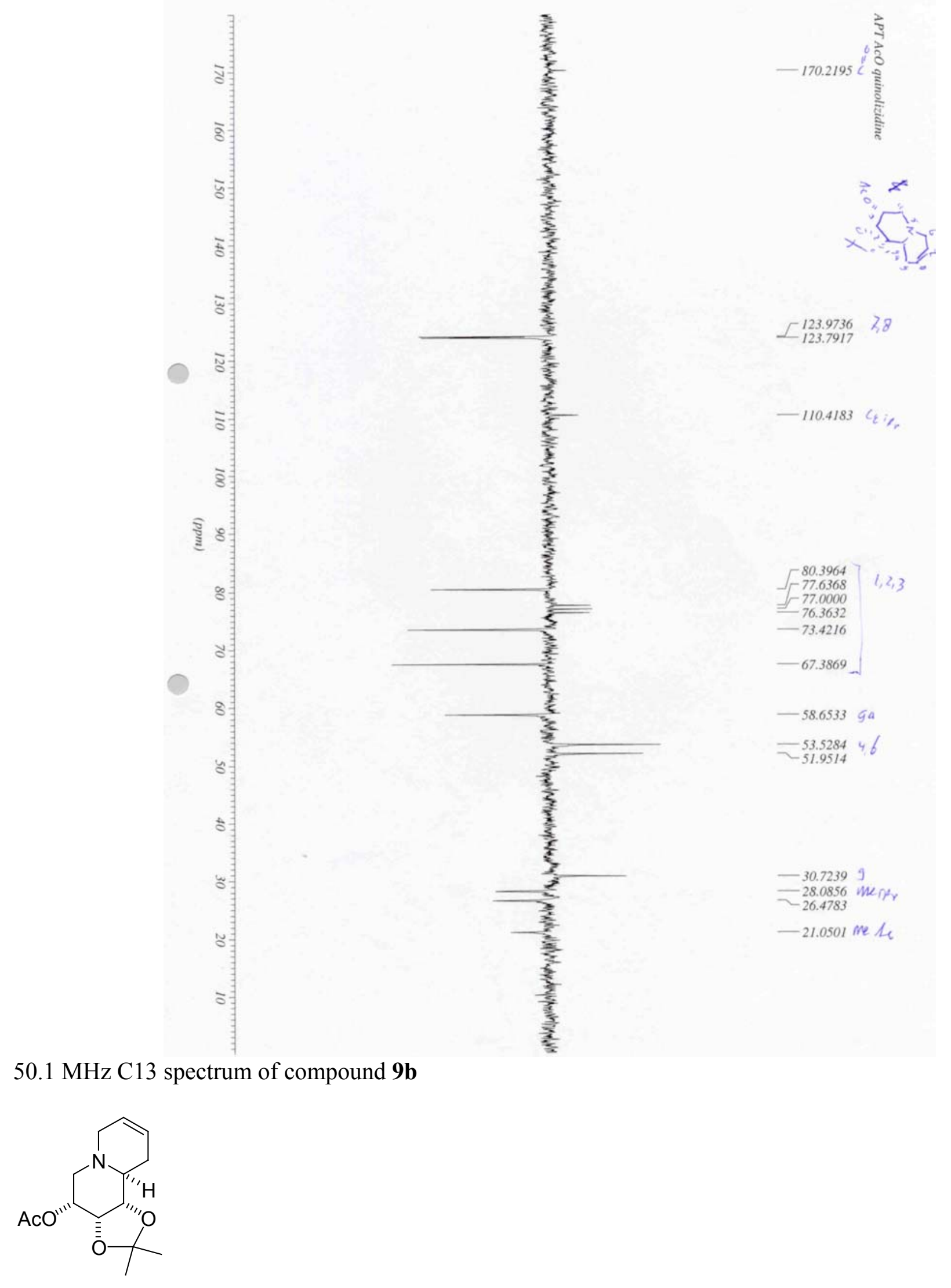




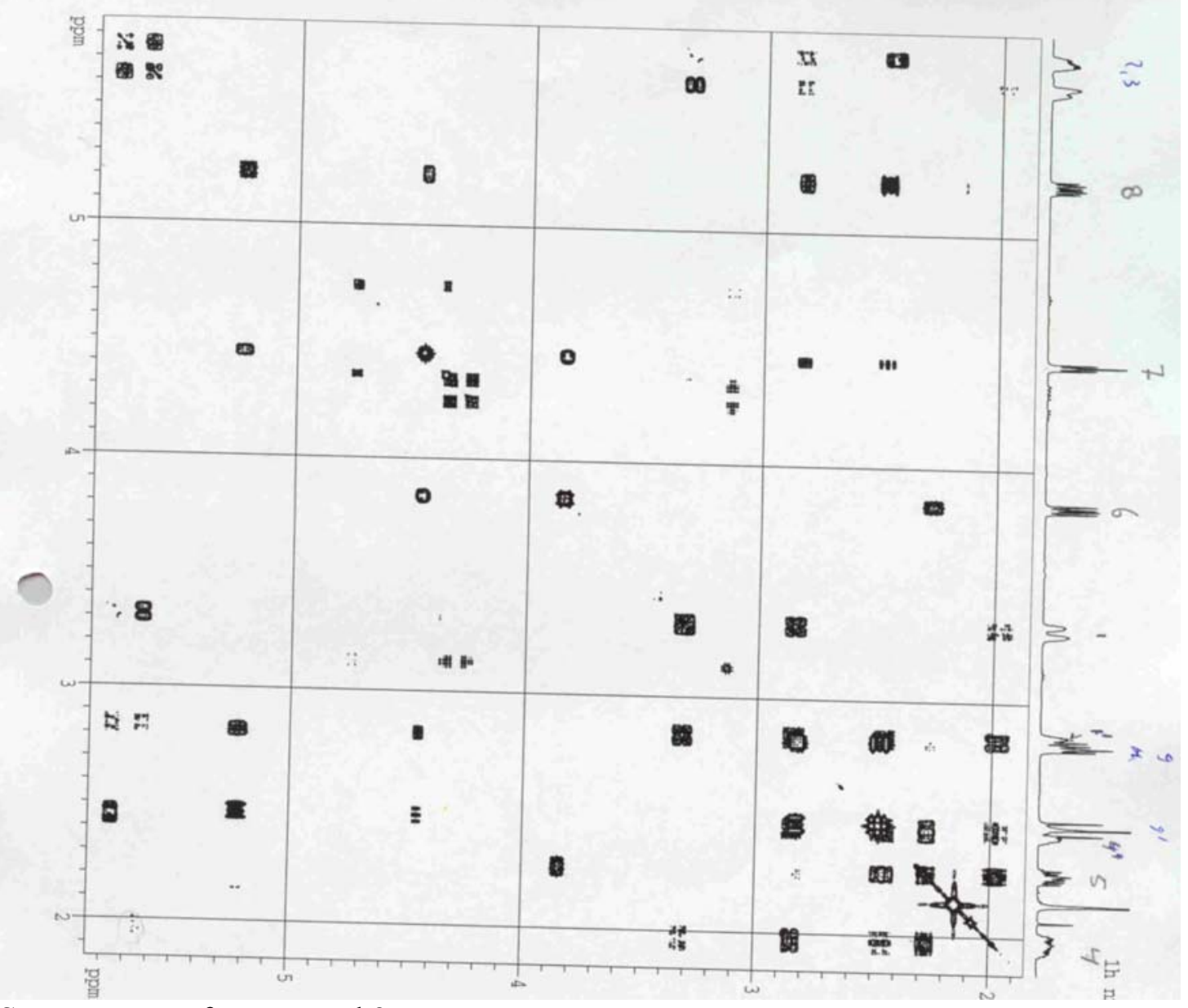

$400 \mathrm{MHz}$ COSY spectrum of compound $\mathbf{9 b}$<smiles>CC(=O)O[C@H]1CN2CC=CC[C@H]2[C@H]2OC(C)(C)O[C@@H]12</smiles> 


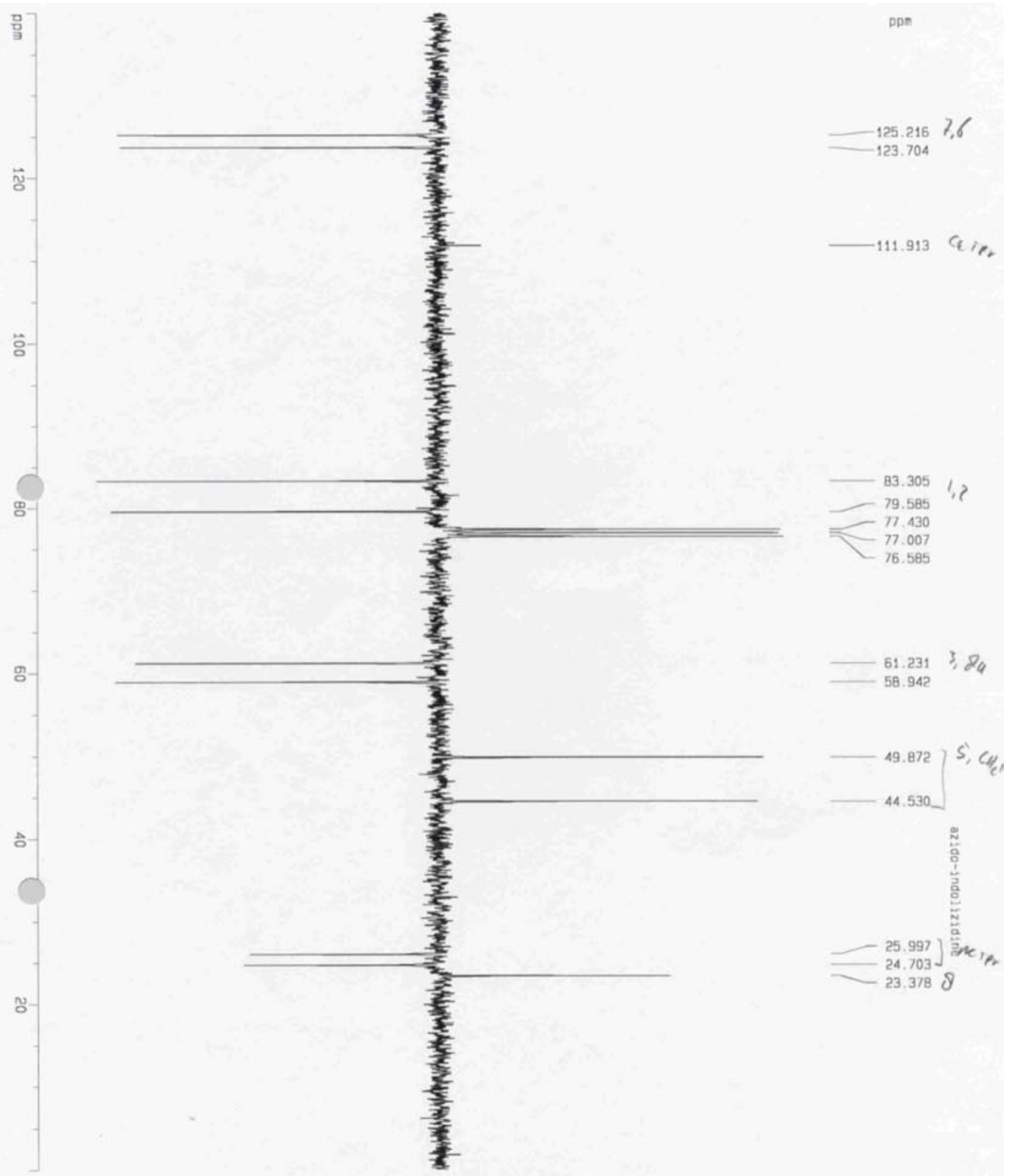

50.1 MHz C13 spectrum of compound $\mathbf{8 c}$<smiles>CC(=O)O[C@H]1CN2CC=CC[C@H]2[C@H]2OC(C)(C)O[C@H]12</smiles> 


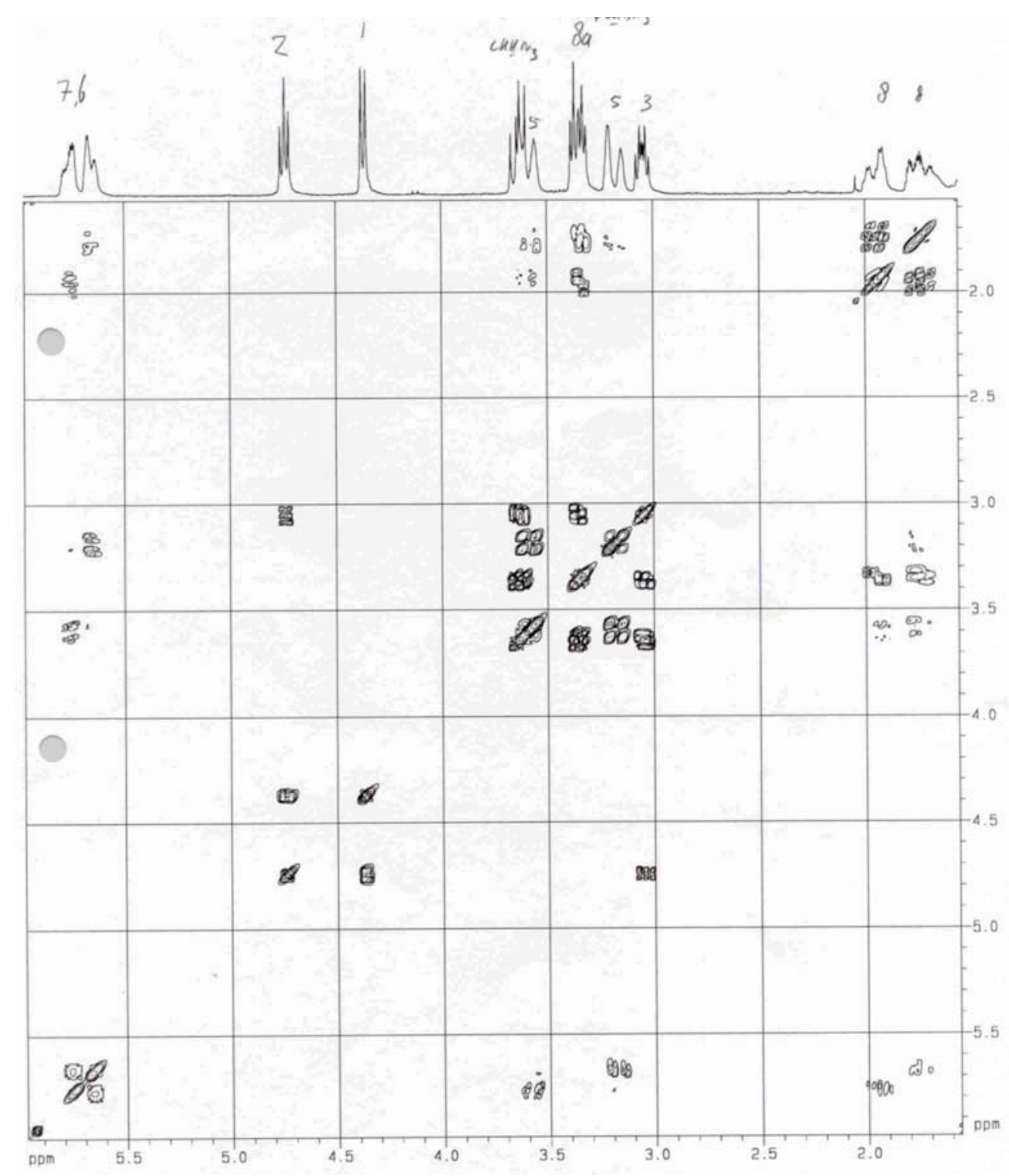

$300 \mathrm{MHz}$ COSY spectrum of compound 8c<smiles>CC(=O)O[C@H]1CN2CC=CC[C@H]2[C@H]2OC(C)(C)O[C@H]12</smiles> 

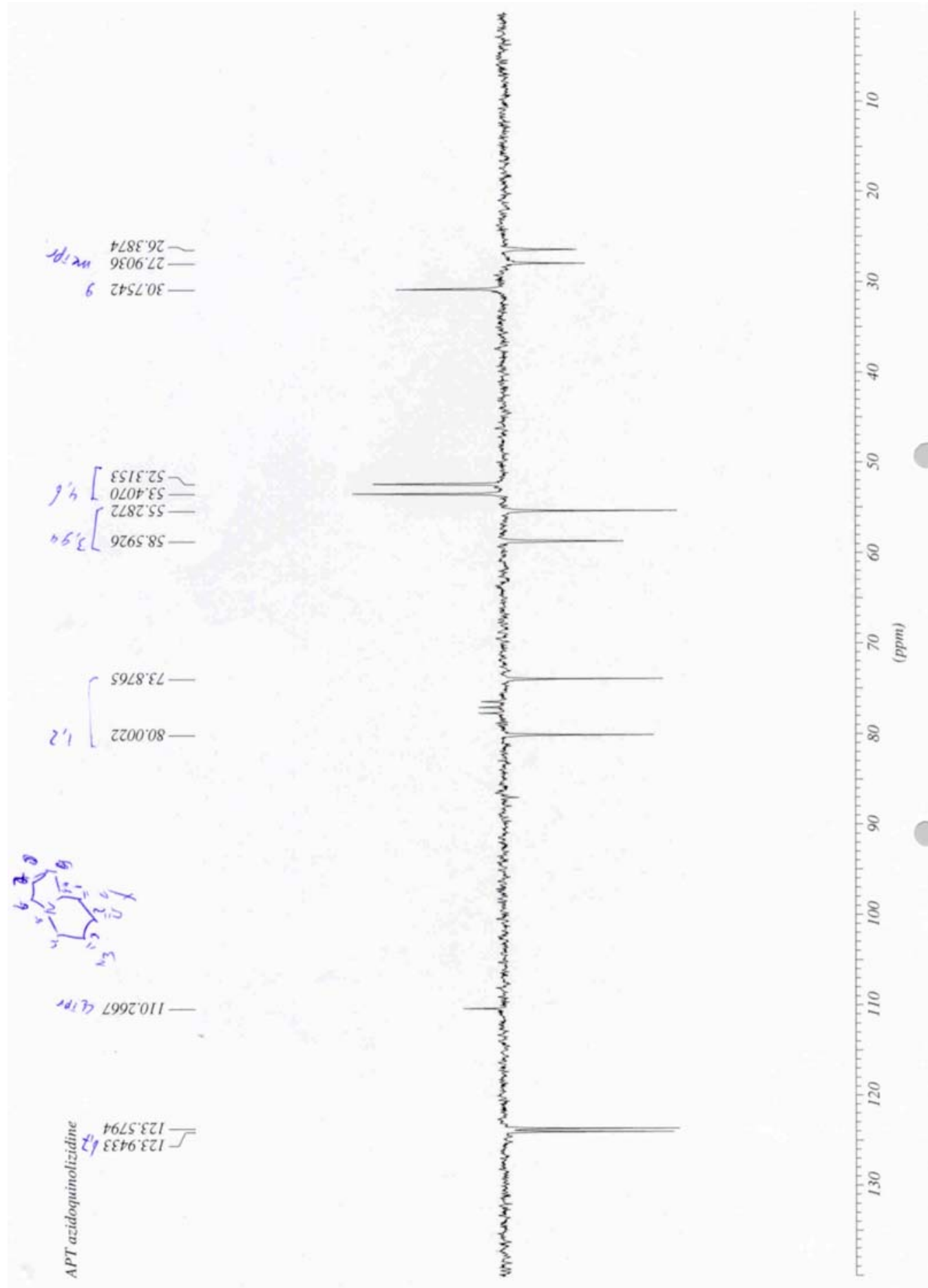

50.1 MHz C13 spectrum of compound 9c<smiles>CC1(C)O[C@H]2[C@H](N)CN3CC=CC[C@H]3[C@H]2O1</smiles> 


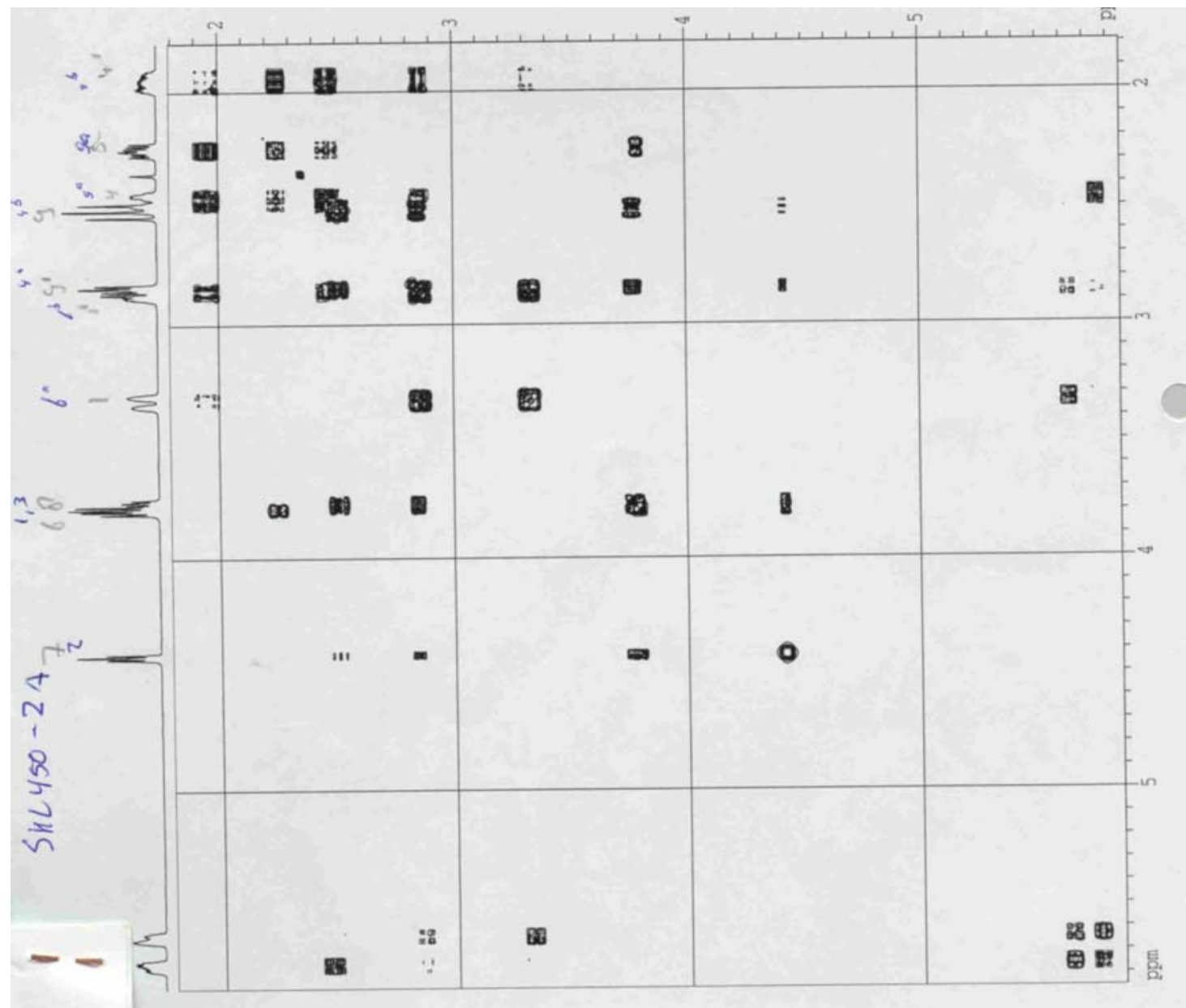

$400 \mathrm{MHz}$ COSY spectrum of compound 9c<smiles>CC1(C)O[C@H]2[C@@H]3CC=CCN3C[C@H](N)[C@H]2O1</smiles> 


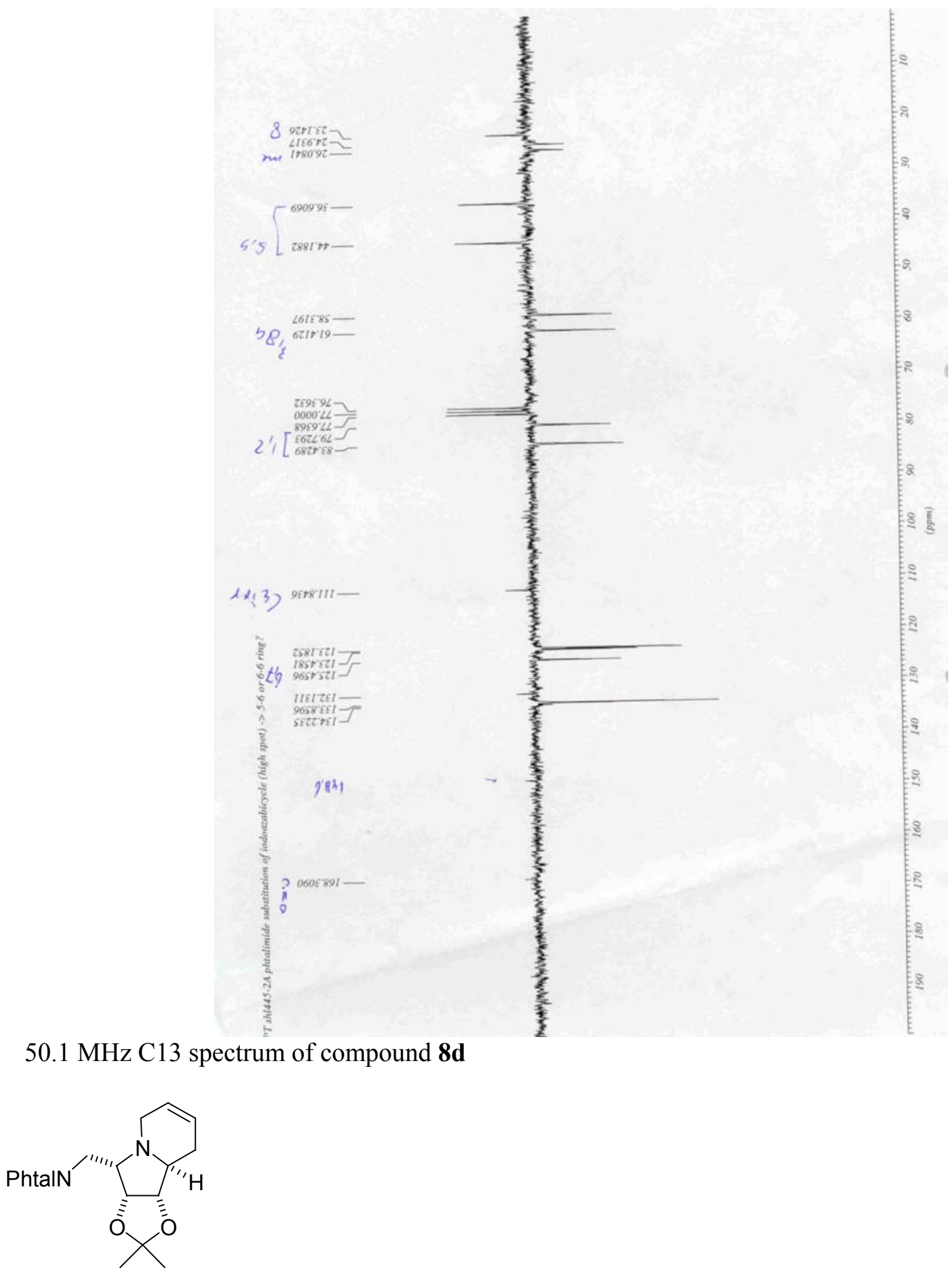




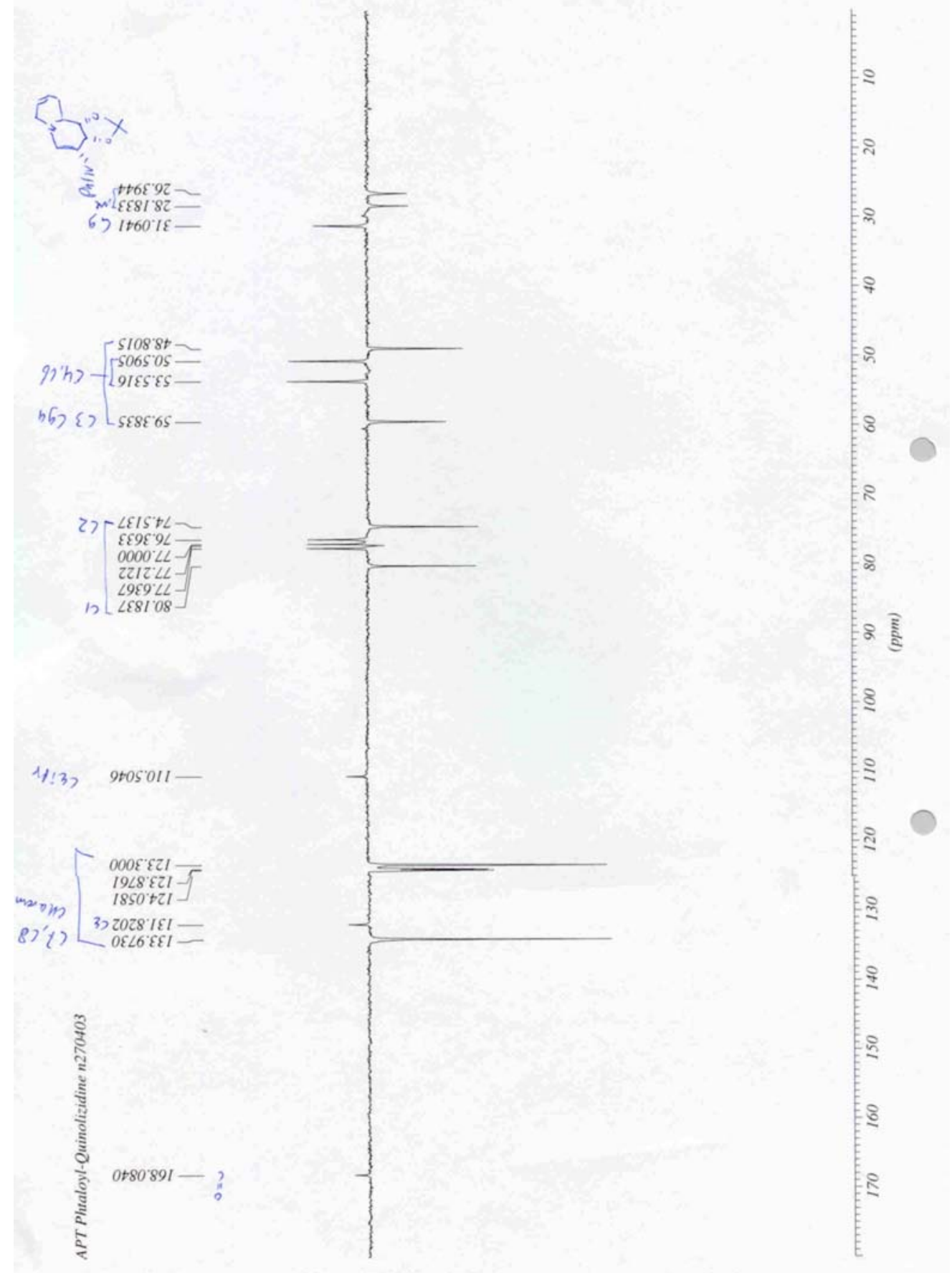

50.1 MHz C13 spectrum of compound 9d<smiles>CC1(C)O[C@H]2[C@H](Nc3ccccc3)CN3CC=CC[C@H]3[C@H]2O1</smiles> 